\title{
Existence of Positive Solutions for $p$-Laplacian Dynamic Equations with Derivative on Time Scales
}

\author{
Jinjun Fan and Liqing Li \\ School of Mathematical Science, Shandong Normal University, Jinan 250014, China \\ Correspondence should be addressed to Jinjun Fan; fjj18@126.com
}

Received 26 November 2012; Accepted 16 January 2013

Academic Editor: Yansheng Liu

Copyright (C) 2013 J. Fan and L. Li. This is an open access article distributed under the Creative Commons Attribution License, which permits unrestricted use, distribution, and reproduction in any medium, provided the original work is properly cited.

We consider the existence of positive solutions of nonlinear p-Laplacian dynamic equations with derivative on time scales. Applying the Avery-Peterson fixed point theorem, we obtain at least three positive solutions to the problem. An example is also presented to illustrate the applications of the obtained results.

\section{Introduction}

In this paper we consider the existence of positive solutions of nonlinear $p$-Laplacian dynamic equations with derivative on time scales

$$
\begin{gathered}
{\left[\phi_{p}\left(u^{\Delta}(t)\right)\right]^{\nabla}+q(t) f\left(t, u(t), u^{\Delta}(t)\right)=0, \quad t \in[0, T]_{\mathbb{T}} ;} \\
\alpha u(0)-\beta u^{\Delta}(0)=\sum_{i=1}^{m-2} u^{\Delta}\left(\xi_{i}\right), \quad u^{\Delta}(T)=0, \quad m \geq 3,
\end{gathered}
$$

where $\mathbb{T}$ is a time scale, $\Delta$ is a delta derivative, $\nabla$ is a nabla derivative, $0<\xi_{1}<\xi_{2}<\xi_{3}<\cdots<\xi_{m-2}<\rho(T), \alpha>0, \beta \geq$ $0,0, T \in \mathbb{T},[0, T]_{\mathbb{T}}=[0, T] \cap \mathbb{T}$, and other types of intervals are defined similarly. Throughout this paper, we denote the $p$-Laplacian operator by $\phi_{p}(u)$; that is, $\phi_{p}(u)=|u|^{p-2} u, p>$ $1,\left(\phi_{p}\right)^{-1}=\phi_{q}$, and $1 / p+1 / q=1$.

The theory of dynamic equations on time scales was introduced firstly by Stefan Hilger in 1988. Since then, more and more scholars are interested in this area. The main reason is that the time scales theory can not only unite continuous and discrete dynamic equations but also have important applications, for example, in the study of insect population models, neural networks, heat transfer, economic, stock market, and epidemic models.

In recent years, there were a lot of attention focused on the study of boundary value problems of nonlinear dynamic equations. Hong in [1] studied the existence of positive solutions for the boundary value problem of $p$-Laplacian dynamic equations

$$
\begin{gathered}
{\left[\phi_{p}\left(u^{\Delta}(t)\right)\right]^{\nabla}+a(t) f(u(t))=0, \quad t \in[0, T]_{T} ;} \\
u(0)-B_{0}\left(u^{\Delta}(\eta)\right)=0, \quad u^{\Delta}(T)=0 .
\end{gathered}
$$

Yaslan in [2] considered nonlinear second-order three-point boundary value problems for dynamic equations on time scales

$$
\begin{gathered}
u^{\Delta \nabla}(t)+h(t) f(t, u(t))=0, \quad t \in\left[t_{1}, t_{3}\right]_{\pi} ; \\
u^{\Delta}\left(t_{3}\right)=0, \quad \alpha u\left(t_{1}\right)-\beta u^{\Delta}\left(t_{1}\right)=u^{\Delta}\left(t_{2}\right) .
\end{gathered}
$$

P. G. Wang and Y. Wang in [3] studied the following boundary value problem:

$$
\begin{gathered}
u^{\Delta \nabla}(t)+a(t) f(u(t))=0, \quad t \in(0, T)_{\mathbb{\Psi}} \\
\beta u(0)-\gamma u^{\Delta}(0)=0, \quad u^{\Delta}(T)=\alpha u(\eta) .
\end{gathered}
$$

Yaslan in [4] considered the existence of solutions of the following $\mathrm{m}$-point boundary value problem on time scales

$$
\begin{gathered}
u^{\Delta \nabla}(t)+h(t) f(t, u(t))=0, \quad t \in\left[t_{1}, t_{m}\right]_{\mathbb{T}} ; \\
u^{\Delta}\left(t_{m}\right)=0, \quad \alpha u\left(t_{1}\right)-\beta u^{\Delta}\left(t_{1}\right)=\sum_{i=2}^{m-1} u^{\Delta}\left(\xi_{i}\right), \quad m \geq 3 .
\end{gathered}
$$


They obtained the existence of at least three nonnegative solutions by using fixed point theorems in cones. The authors in [5-10] dealt with some other classes of differential equations on time scales.

However, the nonlinear terms in the above mentioned literatures [1-4] are not involving a derivative. As far as we know, there is no paper to study the existence of positive solutions for second-order p-Laplacian dynamic equations with derivative on time scales. This paper is to fill this gap. By constructing a special cone and using the Avery-Peterson fixed point theorem, we obtain the existence of at least three nonnegative solutions.

The rest of this paper is organized as follows. In Section 2, we prove several preliminary results which are needed later. In Section 3, conditions for the existence of at least three nonnegative solutions to the BVP (1) are discussed by using the Avery-Peterson fixed point theorem. In Section 4, we give one example to illustrate our results.

\section{Preliminaries and Some Lemmas}

In this section, we first introduce some concepts on time scales and several lemmas.

Let $\mathbb{T}$ be a time scale (an arbitrary nonempty closed subset of the real numbers $R$ ). For $t \in \mathbb{T}$ we define the forward jump operator $\sigma: \mathbb{T} \rightarrow \mathbb{\mathbb { b }}$ by

$$
\sigma(t)=\inf \{s \in \mathbb{T}: s>t\} \quad \text { for } t<\sup \mathbb{T},
$$

while the backward jump operator $\rho: \mathbb{T} \rightarrow \mathbb{T}$ is defined by

$$
\rho(t)=\sup \{s \in \mathbb{T}: s<t\} \quad \text { for } t>\inf \mathbb{T} .
$$

We set $\sigma(M)=M$, if $\mathbb{T}$ has a maximum $M$, and $\rho(m)=m$, if $\mathbb{T}$ has a minimum $m$. If $\sigma(t)>t$, we say that $t$ is right scattered, while we say that $t$ is left scattered, if $\rho(t)<t$. Also, if $t<\sup \mathbb{T}$ and $\sigma(t)=t$, then $t$ is called right dense, and if $t>\inf \mathbb{T}$ and $\rho(t)=t$, then $t$ is called left dense. If $\mathbb{T}$ has a right-scattered minimum $m$, we define $\mathbb{T}_{k}=\mathbb{T}-\{m\}$; otherwise set $\mathbb{T}_{k}=\mathbb{T}$. If $\mathbb{T}$ has a left-scattered maximum $M$, we define $\mathbb{T}^{k}=\mathbb{T}-\{M\}$; otherwise set $\mathbb{T}^{k}=\mathbb{T}$.

For $u: \mathbb{T} \rightarrow R$ and $t \in \mathbb{T}^{k}$, we define the delta derivative of $u(t), u^{\Delta}(t)$, to be the number (provided it exists) with the property that, for any $\varepsilon>0$, there is a neighborhood $U$ of $t$ such that

$$
\left|[u(\sigma(t))-u(s)]-u^{\Delta}(t)[\sigma(t)-s]\right|<\varepsilon|\sigma(t)-s|
$$

for all $s \in U$. For $u: \mathbb{T} \rightarrow R$ and $t \in \mathbb{T}_{k}$, we define the nabla derivative of $u(t), u^{\nabla}(t)$, to be the number (provided it exists) with the property that, for any $\varepsilon>0$, there is a neighborhood $U$ of $t$ such that

$$
\left|[u(\rho(t))-u(s)]-u^{\nabla}(t)[\rho(t)-s]\right|<\varepsilon|\rho(t)-s|
$$

for all $s \in U$.

A function $u: \mathbb{T} \rightarrow R$ is left-dense continuous or ld-continuous provided it is continuous at left-dense points in $\mathbb{T}$, and its right-sided limits exist (finite) at right-dense points in $\mathbb{T}$.
For more details about the subject of concepts and calculations on time scales, please see the book by Bohner and Peterson [11].

Consider the Banach space $E=C_{\mathrm{ld}}^{1}\left([0, \sigma(T)]_{\mathbb{T}} \rightarrow R\right)$; that is,

$E=\left\{u:[0, \sigma(T)]_{\mathbb{T}} \rightarrow R, u\right.$ is $\Delta$ differential on $[0, \sigma(T)]_{\mathbb{T}}$, and $u^{\Delta}$ is ld-continuous on $\left.[0, T]_{\mathbb{V}}\right\}$ with the norm

$$
\|u\|=\max \left\{\sup _{t \in[0, \sigma(T)]_{\mathbb{T}}}|u(t)|, \sup _{t \in[0, T]_{\mathbb{T}}}\left|u^{\Delta}(t)\right|\right\} .
$$

Let

$$
\begin{aligned}
P=\{ & u \in E: u(t) \geq 0, t \in[0, \sigma(T)]_{\mathbb{T}}, \\
& u^{\Delta \nabla}(t) \leq 0, u^{\Delta}(t) \geq 0, t \in[0, T]_{\mathbb{T}}, \\
& \left.\alpha u(0)-\beta u^{\Delta}(0)=\sum_{i=1}^{m-2} u^{\Delta}\left(\xi_{i}\right), m \geq 3\right\} .
\end{aligned}
$$

It is easy to see that $P \subset E$ is a cone in $E$.

For convenience, we list the following assumptions.

$\left(H_{1}\right) f \in C([0, T] \times[0, \infty) \times(-\infty,+\infty),[0, \infty))$ does not vanish identically on any closed subinterval of $[0, T]_{\mathbb{V}}$.

$\left(H_{2}\right)$ The function $q: \mathbb{T} \rightarrow[0, \infty)$ is left-dense continuous, and $0<\int_{0}^{T} q(s) \nabla s<\infty$.

A function $u: \mathbb{T} \rightarrow R$ is said to be a solution of the problem (1) provided that $u$ is delta differential, $u^{\Delta}$ and $\left(\phi_{p}\left(u^{\Delta}\right)\right)^{\nabla}$ are continuous on $T^{k} \cap T_{k}$, and $u$ satisfies the problem (1).

To obtain our main results, we make use of the following lemmas.

Lemma 1. Assume that $\left(H_{1}\right)$ and $\left(H_{2}\right)$ are satisfied. Then $u(t)$ is the solution of the BVP (1) if and only if

$$
\begin{aligned}
u(t)= & \frac{\beta}{\alpha} \phi_{q}\left(\int_{0}^{T} q(s) f\left(s, u(s), u^{\Delta}(s)\right) \nabla s\right) \\
& +\frac{1}{\alpha} \sum_{i=1}^{m-2} \phi_{q}\left(\int_{\xi_{i}}^{T} q(s) f\left(s, u(s), u^{\Delta}(s)\right) \nabla s\right) \\
& +\int_{0}^{t} \phi_{q}\left(\int_{s}^{T} q(\tau) f\left(\tau, u(\tau), u^{\Delta}(\tau)\right) \nabla \tau\right) \triangle s .
\end{aligned}
$$

Proof. Firstly, we prove the necessity. Let $u(t)$ be the solution of the BVP (1). $\nabla$-integrating on (1) from $t$ to $T$, we have

$$
\phi_{p}\left(u^{\Delta}(T)\right)-\phi_{p}\left(u^{\Delta}(t)\right)=-\int_{t}^{T} q(s) f\left(s, u(s), u^{\Delta}(s)\right) \nabla s .
$$

By using the second boundary condition, we get

$$
u^{\Delta}(t)=\phi_{q}\left(\int_{t}^{T} q(s) f\left(s, u(s), u^{\Delta}(s)\right) \nabla s\right) .
$$


$\triangle$-integrating from 0 to $t$, we get

$$
u(t)-u(0)=\int_{0}^{t} \phi_{q}\left(\int_{s}^{T} q(\tau) f\left(\tau, u(\tau), u^{\Delta}(\tau)\right) \nabla \tau\right) \triangle s .
$$

Therefore,

$$
\begin{aligned}
u(t) & =u(0)+\int_{0}^{t} \phi_{q}\left(\int_{s}^{T} q(\tau) f\left(\tau, u(\tau), u^{\Delta}(\tau)\right) \nabla \tau\right) \Delta s, \\
u^{\triangle}(0) & =\phi_{q}\left(\int_{0}^{T} q(s) f\left(s, u(s), u^{\triangle}(s)\right) \nabla s\right), \\
u^{\triangle}\left(\xi_{i}\right) & =\phi_{q}\left(\int_{\xi_{i}}^{T} q(s) f\left(s, u(s), u^{\triangle}(s)\right) \nabla s\right) .
\end{aligned}
$$

Submitting $u^{\Delta}(0), u^{\Delta}\left(\xi_{i}\right)$ into the first boundary condition of the problem (1), we obtain

$$
\begin{aligned}
u(0)= & \frac{\beta}{\alpha} \phi_{q}\left(\int_{0}^{T} q(s) f\left(s, u(s), u^{\Delta}(s)\right) \nabla s\right) \\
& +\frac{1}{\alpha} \sum_{i=1}^{m-2} \phi_{q}\left(\int_{\xi_{i}}^{T} q(s) f\left(s, u(s), u^{\Delta}(s)\right) \nabla s\right) .
\end{aligned}
$$

Thus,

$$
\begin{aligned}
u(t)= & \frac{\beta}{\alpha} \phi_{q}\left(\int_{0}^{T} q(s) f\left(s, u(s), u^{\Delta}(s)\right) \nabla s\right) \\
& +\frac{1}{\alpha} \sum_{i=1}^{m-2} \phi_{q}\left(\int_{\xi_{i}}^{T} q(s) f\left(s, u(s), u^{\Delta}(s)\right) \nabla s\right) \\
& +\int_{0}^{t} \phi_{q}\left(\int_{s}^{T} q(\tau) f\left(\tau, u(\tau), u^{\Delta}(\tau)\right) \nabla \tau\right) \triangle s .
\end{aligned}
$$

Secondly, we prove the sufficiency. Let $u(t)$ be the solution of the problem (12). Then,

$$
\begin{aligned}
& u^{\Delta}(t)=\left[\int_{0}^{t} \phi_{q}\left(\int_{s}^{T} q(\tau) f\left(\tau, u(\tau), u^{\Delta}(\tau)\right) \nabla \tau\right) \Delta s\right]^{\Delta} \\
&=\phi_{q}\left(\int_{t}^{T} q(\tau) f\left(\tau, u(\tau), u^{\Delta}(\tau)\right) \nabla \tau\right) \\
& \phi_{p}\left(u^{\Delta}(t)\right)=\int_{t}^{T} q(\tau) f\left(\tau, u(\tau), u^{\Delta}(\tau)\right) \nabla \tau
\end{aligned}
$$

Taking the $\nabla$-differentiating for (20), we obtain

$$
\begin{aligned}
{\left[\phi_{p}\left(u^{\Delta}(t)\right)\right]^{\nabla} } & =\left[\int_{t}^{T} q(s) f\left(\tau, u(\tau), u^{\Delta}(\tau)\right) \nabla \tau\right]^{\nabla} \\
& =-q(t) f\left(t, u(t), u^{\Delta}(t)\right),
\end{aligned}
$$

that is,

$$
\left[\phi_{p}\left(u^{\Delta}(t)\right)\right]^{\nabla}+q(t) f\left(t, u(t), u^{\Delta}(t)\right)=0 .
$$

By (12), we have

$$
\begin{aligned}
u(0)= & \frac{\beta}{\alpha} \phi_{q}\left(\int_{0}^{T} q(s) f\left(s, u(s), u^{\Delta}(s)\right) \nabla s\right) \\
& +\frac{1}{\alpha} \sum_{i=1}^{m-2} \phi_{q}\left(\int_{\xi_{i}}^{T} q(s) f\left(s, u(s), u^{\Delta}(s)\right) \nabla s\right), \\
u^{\Delta}(0)= & \phi_{q}\left(\int_{0}^{T} q(s) f\left(s, u(s), u^{\Delta}(s)\right) \nabla s\right), \\
u^{\triangle}\left(\xi_{i}\right)= & \phi_{q}\left(\int_{\xi_{i}}^{T} q(s) f\left(s, u(s), u^{\Delta}(s)\right) \nabla s\right) .
\end{aligned}
$$

Hence,

$$
\alpha u(0)-\beta u^{\triangle}(0)=\sum_{i=1}^{m-2} u^{\triangle}\left(\xi_{i}\right), \quad u^{\triangle}(T)=0 .
$$

The proof is complete.

Define operator $F: P \rightarrow E$ by

$$
\begin{aligned}
(F u)(t)= & \frac{\beta}{\alpha} \phi_{q}\left(\int_{0}^{T} q(s) f\left(s, u(s), u^{\Delta}(s)\right) \nabla s\right) \\
& +\frac{1}{\alpha} \sum_{i=1}^{m-2} \phi_{q}\left(\int_{\xi_{i}}^{T} q(s) f\left(s, u(s), u^{\Delta}(s)\right) \nabla s\right) \\
& +\int_{0}^{t} \phi_{q}\left(\int_{s}^{T} q(\tau) f\left(\tau, u(\tau), u^{\Delta}(\tau)\right) \nabla \tau\right) \triangle s .
\end{aligned}
$$

By using Lemma $1, u(t)$ is the solution of the BVP (1) if and only if $u(t)=(F u)(t)$.

Lemma 2. Assume that $\left(H_{1}\right)$ and $\left(H_{2}\right)$ hold. Then $F: P \rightarrow P$ is a completely continuous operator.

Proof. Firstly we prove $F: P \rightarrow P$.

For $u \in P$, by Lemma $1,\left(H_{1}\right)$, and $\left(H_{2}\right)$, we obtain

$$
(F u)(t) \geq 0, \quad t \in[0, \sigma(T)]_{\mathbb{T}},
$$

$$
\begin{aligned}
(F u)^{\Delta}(t) & =\phi_{q}\left(\int_{t}^{T} q(s) f\left(\tau, u(\tau), u^{\Delta}(\tau)\right) \nabla \tau\right) \geq 0, \\
& (F u)^{\Delta \nabla}(t) \leq 0, \\
\alpha(F u)(0)-\beta(F u)^{\Delta}(0) & \\
= & \sum_{i=1}^{m-2} \phi_{q}\left(\int_{\xi_{i}}^{T} q(s) f\left(s, u(s) u^{\Delta}(s)\right) \nabla s\right) \\
= & \sum_{i=1}^{m-2}(F u)^{\Delta}\left(\xi_{i}\right),
\end{aligned}
$$

which implies $F u \in P$. 
Secondly, $F$ maps a bounded set into a bounded set. Let $\overline{P_{c}}=\{u \in P:\|u\| \leq c\}$, where $c>0$. Because $f(t, u, v)$ is continuous, there exists a $C>0$ such that

$$
f(t, u, v) \leq \phi_{p}(C), \quad(t, u, v) \in[0, T]_{\mathbb{T}} \times[0, c] \times[-c, c] .
$$

Hence, for $t \in[0, T]_{\mathbb{T}}, u \in \overline{P_{c}}$, we have

$$
\begin{aligned}
\left|(F u)^{\Delta}(t)\right|= & \left|\phi_{q}\left(\int_{t}^{T} q(s) f\left(\tau, u(\tau), u^{\Delta}(\tau)\right) \nabla \tau\right)\right| \\
< & C \phi_{q}\left(\int_{0}^{T} q(s) \nabla \tau\right)<+\infty, \\
|(F u)(t)|= & \mid \frac{\beta}{\alpha} \phi_{q}\left(\int_{0}^{T} q(s) f\left(s, u(s), u^{\Delta}(s)\right) \nabla s\right) \\
& +\frac{1}{\alpha} \sum_{i=1}^{m-2} \phi_{q}\left(\int_{\xi_{i}}^{T} q(s) f\left(s, u(s), u^{\Delta}(s)\right) \nabla s\right) \\
& +\int_{0}^{t} \phi_{q}\left(\int_{s}^{T} q(\tau) f\left(\tau, u(\tau), u^{\Delta}(\tau)\right) \nabla \tau\right) \triangle s \mid \\
\leq & \frac{C(\beta+m-2+\alpha T)}{\alpha} \phi_{q}\left(\int_{0}^{T} q(s) \nabla s\right)<+\infty .
\end{aligned}
$$

Consequently, $F \overline{P_{c}}$ is bounded.

For $t_{1}, t_{2} \in[0, T]_{\mathbb{T}}, u \in \overline{P_{c}}$, we get

$$
\begin{aligned}
\mid(F u) & \left(t_{1}\right)-(F u)\left(t_{2}\right) \mid \\
& =\left|\int_{t_{1}}^{t_{2}} \phi_{q}\left(\int_{s}^{T} q(\tau) f\left(\tau, u(\tau), u^{\Delta}(\tau)\right) \nabla \tau\right) \Delta s\right| \\
& \leq C\left|\int_{t_{1}}^{t_{2}} \phi_{q}\left(\int_{s}^{T} q(\tau) \nabla \tau\right) \Delta s\right| \\
& \leq C\left|t_{1}-t_{2}\right| \phi_{q}\left(\int_{0}^{T} q(\tau) \nabla \tau\right) \longrightarrow 0, \quad t_{1} \longrightarrow t_{2} .
\end{aligned}
$$

Thus $F \overline{P_{c}}$ is relatively compact by using the Arzela-Ascoli theorem.

Finally we prove that $F: \overline{P_{c}} \rightarrow P$ is continuous. Let $\left\{u_{n}\right\}_{n=1}^{\infty} \subset \bar{P}_{c}$ satisfying

$$
\lim _{n \rightarrow \infty}\left\|u_{n}-u_{0}\right\|=0
$$

This means that

$$
\lim _{n \rightarrow \infty}\left|u_{n}-u_{0}\right|=0, \quad \lim _{n \rightarrow \infty}\left|u_{n}^{\Delta}-u_{0}^{\Delta}\right|=0 .
$$

Note that

$$
\begin{aligned}
\left(F u_{n}\right)(t)= & \frac{\beta}{\alpha} \phi_{q}\left(\int_{0}^{T} q(s) f\left(s, u_{n}(s), u_{n}^{\Delta}(s)\right) \nabla s\right) \\
& +\frac{1}{\alpha} \sum_{i=1}^{m-2} \phi_{q}\left(\int_{\xi_{i}}^{T} q(s) f\left(s, u_{n}(s), u_{n}^{\Delta}(s)\right) \nabla s\right), \\
\left(F u_{n}\right)^{\Delta}(t)= & \phi_{q}\left(\int_{t}^{T} q(s) f\left(\tau, u_{n}(\tau), u_{n}^{\Delta}(\tau)\right) \nabla \tau\right) .
\end{aligned}
$$

Using the Lebesgue dominated convergence theorem on time scales, this together with (28) implies that

$$
\begin{aligned}
\lim _{n \rightarrow \infty}\left(F u_{n}\right)(t)= & \frac{\beta}{\alpha} \phi_{q}\left(\int_{0}^{T} q(s) f\left(s, u_{0}(s), u_{0}^{\Delta}(s)\right) \nabla s\right) \\
& +\frac{1}{\alpha} \sum_{i=1}^{m-2} \phi_{q}\left(\int_{\xi_{i}}^{T} q(s) f\left(s, u_{0}(s), u_{0}^{\Delta}(s)\right) \nabla s\right), \\
\lim _{n \rightarrow \infty}\left(F u_{n}\right)^{\Delta}(t)= & \phi_{q}\left(\int_{t}^{T} q(s) f\left(\tau, u_{0}(\tau), u_{0}^{\Delta}(\tau)\right) \nabla \tau\right) .
\end{aligned}
$$

From the definition of $F$, we know that

$$
\lim _{n \rightarrow \infty}\left(F u_{n}\right)(t)=\left(F u_{0}\right)(t) .
$$

Therefore $F$ is continuous on $\overline{P_{c}}$. The proof is complete.

Lemma 3. If $u \in P$, then

(i) $u(t) \geq(t / \sigma(T)) u(\sigma(T)), t \in[0, \sigma(T)]_{\mathbb{T}}$;

(ii) $s u(t) \leq t u(s), s, t \in[0, \sigma(T)]_{\mathbb{T}}$ and $s \leq t$.

Proof. First, prove (i). Let $g(t)=u(t)-(t / \sigma(T)) u(\sigma(T))$. From $g^{\Delta \nabla}(t)=u^{\Delta \nabla}(t) \leq 0, g(0)=u(0) \geq 0$, and $g(\sigma(T))=0$, we have $g(t) \geq 0$.

Second, prove (ii). Since $u(t)$ is a concave function, for $0<s \leq t \leq \sigma(T)$, we have

$$
\begin{gathered}
\frac{u(t)-u(0)}{t} \leq \frac{u(s)-u(0)}{s}, \\
s u(t)-s u(0) \leq t u(s)-t u(0), \\
s u(t) \leq t u(s)+(s-t) u(0), \\
s u(t)+(t-s) u(0) \leq t u(s)
\end{gathered}
$$

From $(t-s) u(0) \geq 0$, it follows that $s u(t) \leq t u(s)$. The proof is complete.

Lemma 4. Let $u \in P$. Then there exists a real number $M>0$ such that $\sup _{t \in[0, \sigma(T)]_{T}} u(t) \leq M \sup _{t \in[0, T]_{T}} u^{\Delta}(t)$, where $M=$ $\max \{(\beta+m-2) \sigma(T) / \alpha T+\sigma(T), 1\}$. 
Proof. Since $u(t)=u(0)+\int_{0}^{t} u^{\Delta}(s) \Delta s, u^{\Delta}(t) \geq 0$, and $u(0)=$ $(1 / \alpha) \sum_{i=1}^{m-2} u^{\Delta}\left(\xi_{i}\right)+(\beta / \alpha) u^{\Delta}(0)$, we have

$$
\begin{aligned}
u(T) & =\sup _{t \in[0, T]_{T}} u(t) \\
& =\sup _{t \in[0, T]_{\pi}}\left\{\frac{1}{\alpha} \sum_{i=1}^{m-2} u^{\Delta}\left(\xi_{i}\right)+\frac{\beta}{\alpha} u^{\Delta}(0)+\int_{0}^{t} u^{\Delta}(s) \Delta s\right\} \\
& \leq \frac{m-2}{\alpha} \sup _{t \in[0, T]_{T}} u^{\Delta}(t)+\frac{\beta}{\alpha} \sup _{t \in[0, T]_{T}} u^{\Delta}(t)+\sup _{t \in[0, T]_{T}} T u^{\Delta}(t) \\
& =\left(\frac{\beta+m-2}{\alpha}+T\right) \sup _{t \in[0, T]_{T}} u^{\Delta}(t) .
\end{aligned}
$$

By Lemma 3, we obtain

$$
\begin{aligned}
\sup _{t \in[0, \sigma(T)]_{T}} u(t) & =u(\sigma(T)) \leq \frac{u(T)}{T} \sigma(T) \\
& \leq \frac{\sigma(T)}{T}\left(\frac{\beta+m-2}{\alpha}+T\right) \sup _{t \in[0, T]_{T}} u^{\Delta}(t) \\
& \leq M \sup _{t \in[0, T]_{T}} u^{\Delta}(t) .
\end{aligned}
$$

The proof is complete.

In the rest of this section, we state the main tool used in this paper.

Let $\gamma, \theta, \alpha$, and $\psi$ be functionals on cone $P$ in a real Banach space, where $\gamma, \theta$ are nonnegative continuous convex functionals, $\alpha$ is a nonnegative continuous concave functional, $\psi$ is a nonnegative continuous functional, and $a, b, c, d \geq 0$; define the following sets:

$$
\begin{aligned}
& P(\gamma, d)=\{x \in P: \gamma(x)<d\}, \\
& P(\gamma, \alpha, b, d)=\{x \in P: b \leq \alpha(x), \gamma(x) \leq d\}, \\
& P(\gamma, \theta, \alpha, b, c, d)=\{x \in P: b \leq \alpha(x), \\
& \theta(x) \leq c, \gamma(x) \leq d\}, \\
& R(\gamma, \psi, a, d)=\{x \in P: a \leq \psi(x), \gamma(x) \leq d\} .
\end{aligned}
$$

Lemma 5 (see [1], the Avery-Peterson fixed point theorem). Let $P$ be a cone in a real Banach space $E$ and $\gamma, \theta, \psi$, and $\alpha$ defined as above; moreover, $\psi$ satisfies $\psi(\lambda x) \leq \lambda \psi(x)$ for $0 \leq$ $\lambda \leq 1$, and

$$
\alpha(x) \leq \psi(x), \quad\|x\| \leq M \gamma(x), \quad \forall x \in \overline{P(\gamma, d)}
$$

for some positive numbers $M$ and $d$. Suppose that $F$ : $\overline{P(\gamma, d)} \rightarrow \overline{P(\gamma, d)}$ is a completely continuous operator and that there exist positive real numbers $a, b$, and $c$ with $a<b$ such that the following conditions are satisfied:

(i) $\{x \in P(\gamma, \theta, \alpha, b, c, d): \alpha(x)>b\} \neq \emptyset$, and $\alpha(F(x))>b$ for $x \in P(\gamma, \theta, \alpha, b, c, d)$; (ii) $\alpha(F(x))>b$ for $x \in P(\gamma, \alpha, b, d)$ with $\theta(F(x))>c$;

(iii) $0 \bar{\epsilon} R(\gamma, \psi, a, d)$ and $\psi(F(x))<a$ for $x \in R(\gamma, \psi$, $a, d)$ with $\psi(x)=a$.

Then $F$ has at least three fixed points $x_{1}, x_{2}$, and $x_{3} \in$ $\overline{P(\gamma, d)}$ such that

$$
\begin{aligned}
& \gamma\left(x_{i}\right) \leq d \text { for } i=1,2,3 \\
& b<\alpha\left(x_{1}\right) \\
& a<\psi\left(x_{2}\right) \text { with } \alpha\left(x_{2}\right)<b ; \\
& \psi\left(x_{3}\right)<a .
\end{aligned}
$$

\section{Main Results}

Define the nonnegative continuous convex functionals $\gamma$ and $\theta$, nonnegative continuous concave functional $\alpha$, and nonnegative continuous functional $\psi$ as follows:

$$
\begin{gathered}
\gamma(u)=\sup _{t \in[0, T]_{T}} u^{\Delta}(t)=u^{\Delta}(0), \\
\theta(u)=\sup _{t \in[0, T]_{T}} u(t)=u(T), \\
\alpha(u)=\inf _{t \in\left[\xi_{m-2}, T\right]_{T}} u(t)=u\left(\xi_{m-2}\right), \\
\psi(u)=\sup _{t \in[0, T]_{T}} u(t)=u(T) .
\end{gathered}
$$

By Lemma 3, we have

$$
\begin{aligned}
\|u\| & =\max \left\{\sup _{t \in[0, \sigma(T)]_{\top}}|u(t)|, \sup _{t \in[0, T]_{\top}}\left|u^{\Delta}(t)\right|\right\} \\
& \leq M \gamma(u), \quad u \in P .
\end{aligned}
$$

Define

$$
\begin{aligned}
& A:=\phi_{q}\left(\int_{0}^{T} q(s) \nabla s\right) \\
& B:=\frac{\beta+m-2+\alpha \xi_{m-2}}{\alpha} \phi_{q}\left(\int_{\xi_{m-2}}^{T} q(\tau) \nabla \tau\right), \\
& C:=\frac{\beta+m-2+\alpha T}{\alpha} \phi_{q}\left(\int_{0}^{T} q(s) \nabla s\right) \\
& l:=\frac{\xi_{m-2}(\alpha T+\beta+m-2)}{\alpha T}
\end{aligned}
$$

Theorem 6. Assume that $\left(H_{1}\right)$ and $\left(H_{2}\right)$ hold. In addition, suppose that there exist constants $a, b$, and $d$ such that $0<a<$ $b \leq l d$ and the function $f$ satisfies the following conditions:

$\left(A_{1}\right) f(t, u, v) \leq \phi_{p}(d / A),(t, u, v) \in[0, T]_{\mathbb{T}} \times[0, M d] \times$ $[-d, d]$

$\left(A_{2}\right) f(t, u, v)>\phi_{p}(b / B),(t, u, v) \in\left[\xi_{m-2}, T\right]_{\mathbb{T}} \times$ $\left[b, b T / \xi_{m-2}\right] \times[-d, d]$ 
$\left(A_{3}\right) f(t, u, v)<\phi_{p}(a / C),(t, u, v) \in[0, T]_{\mathbb{T}} \times[0, a] \times$ $[-d, d]$.

Then BVP (1) has at least three nonnegative solutions $u_{1}, u_{2}$, and $u_{3}$ satisfying

(i) $\gamma\left(u_{i}\right) \leq d, i=1,2,3$;

(ii) $\alpha\left(u_{1}\right)>b, a<\psi\left(u_{2}\right), \alpha\left(u_{2}\right)<b$, and $\psi\left(u_{3}\right)<a$.

Proof. It is sufficient to show that $F$ has at least three fixed points. To this purpose, we show that all conditions of Lemma 5 are fulfilled. Now we divide this proof into three steps.

Step 1. Consider, $F: \overline{P(\gamma, d)} \rightarrow \overline{P(\gamma, d)}$.

For $u \in \overline{P(\gamma, d)}$, we have $\gamma(u)=\sup _{t \in[0, T]_{T}} u^{\Delta}(t) \leq d$. By Lemma 4 , we get $\sup _{t \in[0, \sigma(T)]_{T}} u(t) \leq M d$. From $\left(A_{1}\right)$, it follows that

$$
\begin{aligned}
\gamma(F u) & =\sup _{t \in[0, T]_{T}}(F u)^{\Delta}(t)=(F u)^{\Delta}(0) \\
& =\phi_{q}\left(\int_{0}^{T} q(s) f\left(s, u(s), u^{\Delta}(s)\right) \nabla s\right) \\
& \leq \frac{d}{A} \phi_{q}\left(\int_{0}^{T} q(s) \nabla s\right)=d .
\end{aligned}
$$

Applying this and Lemma 2, we have that $F: \overline{P(\gamma, d)} \rightarrow$ $\overline{P(\gamma, d)}$ is completely continuous.

Step 2. (i) and (ii) in Lemma 5 hold.

Let $u(t)=b T(\alpha t+\beta+m-2) /(\beta+m-2+\alpha T) \xi_{m-2}, t \in$ $[0, T]_{\mathbb{T}}$. Clearly $u(t) \geq 0, u^{\Delta \nabla}(t) \leq 0$, and

$$
\begin{aligned}
\alpha u(0)-\beta u^{\Delta}(0)= & \frac{\alpha b T(\beta+m-2)}{(\beta+m-2+\alpha T) \xi_{m-2}} \\
& -\frac{\beta \alpha b T}{(\beta+m-2+\alpha T) \xi_{m-2}} \\
= & \frac{\alpha b T(m-2)}{(\beta+m-2+\alpha T) \xi_{m-2}} \\
= & \sum_{i=1}^{m-2} u^{\Delta}\left(\xi_{i}\right) .
\end{aligned}
$$

So $u \in P$.

From

$$
\begin{gathered}
\alpha(u)=u\left(\xi_{m-2}\right)=\frac{b T\left(\alpha \xi_{m-2}+\beta+m-2\right)}{(\beta+m-2+\alpha T) \xi_{m-2}}>b, \\
\theta(u)=\frac{b T}{\xi_{m-2}}, \quad \gamma(u)=\frac{b \alpha T}{(\beta+m-2+\alpha T) \xi_{m-2}} \leq d,
\end{gathered}
$$

we have $\{u \in P(\gamma, \theta, \alpha, b, c, d): \alpha(u)>b\} \neq \emptyset$, where $c=$ $b T / \xi_{m-2}$.
By $\left(A_{2}\right)$, for $u \in P(\gamma, \theta, \alpha, b, c, d)$, we know

$$
\begin{aligned}
\alpha(F u)= & (F u)\left(\xi_{m-2}\right) \\
= & \frac{\beta}{\alpha} \phi_{q}\left(\int_{0}^{T} q(s) f\left(s, u(s), u^{\Delta}(s)\right) \nabla s\right) \\
& +\frac{1}{\alpha} \sum_{i=1}^{m-2} \phi_{q}\left(\int_{\xi_{i}}^{T} q(s) f\left(s, u(s), u^{\Delta}(s)\right) \nabla s\right) \\
& +\int_{0}^{\xi_{m-2}} \phi_{q}\left(\int_{s}^{T} q(\tau) f\left(\tau, u(\tau), u^{\Delta}(\tau)\right) \nabla \tau\right) \triangle s \\
> & \frac{\beta}{\alpha} \phi_{q}\left(\int_{\xi_{m-2}}^{T} q(s) f\left(s, u(s), u^{\Delta}(s)\right) \nabla s\right) \\
& +\frac{m-2}{\alpha} \phi_{q}\left(\int_{\xi_{m-2}}^{T} q(s) f\left(s, u(s), u^{\Delta}(s)\right) \nabla s\right) \\
& +\int_{0}^{\xi_{m-2}} \phi_{q}\left(\int_{\xi_{m-2}}^{T} q(\tau) f\left(\tau, u(\tau), u^{\Delta}(\tau)\right) \nabla \tau\right) \triangle s \\
> & \frac{\beta+m-2+\alpha \xi_{m-2}}{\alpha} \phi_{q}\left(\int_{\xi_{m-2}}^{T} q(\tau) \nabla \tau\right) \times \frac{b}{B}=b .
\end{aligned}
$$

So (i) in Lemma 5 is satisfied.

For $u \in P(\gamma, \alpha, b, d)$ with $\theta(F u)>b\left(T / \xi_{m-2}\right)$, since $F u \in$ $P$, by (ii) in Lemma 3 , we can get

$$
(F u)\left(\xi_{m-2}\right) \geq \frac{\xi_{m-2}}{T}(F u)(T) .
$$

So

$$
\alpha(F u)=(F u)\left(\xi_{m-2}\right) \geq \frac{\xi_{m-2}}{T}(F u)(T)=\frac{\xi_{m-2}}{T} \theta(F u)>b .
$$

Then (ii) in Lemma 5 holds.

Step 3. (iii) in Lemma 5 holds.

Clearly $\psi(0)=0<a$, so $0 \bar{\epsilon} R(\gamma, \psi, a, d)$. If $u \in$ $R(\gamma, \psi, a, d)$ with $\psi(u)=a$, by $\left(A_{3}\right)$, we obtain

$$
\begin{aligned}
\psi(F u)= & \sup _{t \in(0, T)_{\mathbb{T}}}(F u)(t) \\
= & \frac{\beta}{\alpha} \phi_{q}\left(\int_{0}^{T} q(s) f\left(s, u(s), u^{\Delta}(s)\right) \nabla s\right) \\
& +\frac{1}{\alpha} \sum_{i=1}^{m-2} \phi_{q}\left(\int_{\xi_{i}}^{T} q(s) f\left(s, u(s), u^{\Delta}(s)\right) \nabla s\right) \\
& +\int_{0}^{T} \phi_{q}\left(\int_{s}^{T} q(\tau) f\left(\tau, u(\tau), u^{\Delta}(\tau)\right) \nabla \tau\right) \triangle s \\
\leq & \frac{\beta}{\alpha} \phi_{q}\left(\int_{0}^{T} q(s) f\left(s, u(s), u^{\Delta}(s)\right) \nabla s\right)
\end{aligned}
$$




$$
\begin{aligned}
& +\frac{m-2}{\alpha} \phi_{q}\left(\int_{0}^{T} q(\tau) f\left(\tau, u(\tau), u^{\Delta}(\tau)\right) \nabla \tau\right) \\
& +T \phi_{q}\left(\int_{0}^{T} q(\tau) f\left(\tau, u(\tau), u^{\Delta}(\tau)\right) \nabla \tau\right) \\
& <\frac{\beta+m-2+\alpha T}{\alpha} \phi_{q}\left(\int_{0}^{T} q(\tau) \nabla \tau\right) \cdot \frac{a}{C}=a .
\end{aligned}
$$

From above, the hypotheses of the Avery-Peterson theorem are satisfied. Therefore the problem (1) has at least three nonnegative solutions $u_{1}, u_{2}$, and $u_{3}$ satisfying

(i) $\gamma\left(u_{i}\right) \leq d, i=1,2,3$;

(ii) $\alpha\left(u_{1}\right)>b, a<\psi\left(u_{2}\right), \alpha\left(u_{2}\right)<b$, and $\psi\left(u_{3}\right)<a$.

The proof is complete.

\section{Application}

Let $\mathbb{T}=\{0,1 / 8,1 / 4,3 / 8\} \cup[1 / 2,1] \cup\{9 / 8\}$. We consider the following BVP:

$$
\begin{array}{ll}
\left(u^{\Delta}(t)\right)^{\nabla}+t f\left(t, u(t), u^{\Delta}(t)\right)=0, & t \in[0,1]_{\mathbb{T}}, \\
u(0)-u^{\Delta}(0)=u^{\Delta}\left(\frac{1}{4}\right)+u^{\Delta}\left(\frac{1}{2}\right), & u^{\Delta}(1)=0,
\end{array}
$$

where $q(t)=t, \alpha=\beta=1, \xi_{1}=1 / 4, \xi_{2}=1 / 2, m=4, p=$ 2 , and

$$
f(t, u, v)= \begin{cases}\frac{t}{8}+\frac{49 u^{2}}{256}+\frac{\sin v}{64}, & u \leq 4 \\ \frac{t}{8}+\frac{49}{16}+\frac{\sin v}{64}, & u \geq 4\end{cases}
$$

By a simple calculation, we obtain $M=9 / 2, A=17 / 32, B=$ $21 / 16, C=17 / 8$, and $l=2$. Choose $a=2, b=4$, and $d=$ 10. Thus $f(t, u, v)$ satisfies

(1) $(t, u, v) \in[0,1]_{\mathbb{T}} \times[0,45] \times[-10,10], f(t, u, v)<10 \times$ $(32 / 17)=320 / 17=d / A$,

(2) $(t, u, v) \in[1 / 2,1]_{\mathbb{T}} \times[4,8] \times[-10,10], f(t, u, v)>4 \times$ $(16 / 21)=64 / 21=b / B$,

(3) $(t, u, v) \in[0,1]_{\mathbb{T}} \times[0,2] \times[-10,10], f(t, u, v)<2 \times$ $(8 / 17)=16 / 17=a / C$.

Then all conditions of Theorem 6 hold. Therefore, the problem (51) has at least three nonnegative solutions $u_{1}, u_{2}$, and $u_{3}$ such that

$$
\begin{array}{cc}
u_{i}^{\Delta}(0) \leq 10, & i=1,2,3 ; \\
u_{1}\left(\frac{1}{2}\right)>4 ; & u_{2}(1)>2, \\
u_{2}\left(\frac{1}{2}\right)<4 ; & u_{3}(1)<2 .
\end{array}
$$

\section{Acknowledgments}

The authors express their heartful thanks to reviewer(s) comments. This research is supported by Innovation Project for Graduate Education of Shandong Province (SDYY10058).

\section{References}

[1] S. H. Hong, "Triple positive solutions of three-point boundary value problems for p-Laplacian dynamic equations on time scales," Journal of Computational and Applied Mathematics, vol. 206, no. 2, pp. 967-976, 2007.

[2] I. Yaslan, "Multiple positive solutions for nonlinear threepoint boundary value problems on time scales," Computers and Mathematics with Applications, vol. 55, no. 8, pp. 1861-1869, 2008.

[3] P. G. Wang and Y. Wang, "Existence of positive solutions for second-order m-point boundary value problems on time scales," Acta Mathematica Sinica, vol. 50, no. 3, pp. 701-706, 2007.

[4] I. Yaslan, "Multi-point boundary value problems on time scales," Nonlinear Dynamics and Systems Theory, vol. 10, no. 3, pp. 305-316, 2010.

[5] J. P. Sun, "Twin positive solutions of nonlinear first-order boundary value problems on time scales," Nonlinear Analysis, Theory, Methods and Applications, vol. 68, no. 6, pp. 1754-1758, 2008.

[6] D. R. Anderson and I. Y. Karaca, "Higher-order three-point boundary value problem on time scales," Computers and Mathematics with Applications, vol. 56, no. 9, pp. 2429-2443, 2008.

[7] Y. Li and H. Zhang, "Positive periodic solutions of neutral functional differential equations with state dependent delays on time scales," Acta Mathematica Sinica, vol. 30, no. 3, pp. 730$742,2010$.

[8] Y. Tian and W. Ge, "Existence and uniqueness results for nonlinear first-order three-point boundary value problems on time scales," Nonlinear Analysis, Theory, Methods and Applications, vol. 69, no. 9, pp. 2833-2842, 2008.

[9] J. Fan, X. Zhang, and Y. Liu, "Existence of positive solutions of the $\mathrm{m}$-point boundary value problem with p-Laplace operator on time scales," Journal of Shandong University, vol. 47, no. 6, pp. 16-19, 2012 (Chinese).

[10] J. Fan, X. Zhang, and Y. Liu, "Positive solutions of nonlinear $\mathrm{m}$-point bound- ary value problem with $\mathrm{p}$-Laplace operator on time scales," Journal of University of Jinan, vol. 26, no. 4, pp. 415418, 2012 (Chinese).

[11] M. Bohner and A. Peterson, Advances in Dynamic Equations on Time Scales, Birkhäauser, Boston, Mass, USA, 2003. 


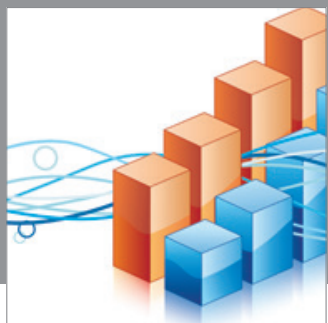

Advances in

Operations Research

mansans

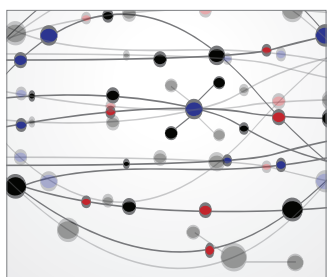

The Scientific World Journal
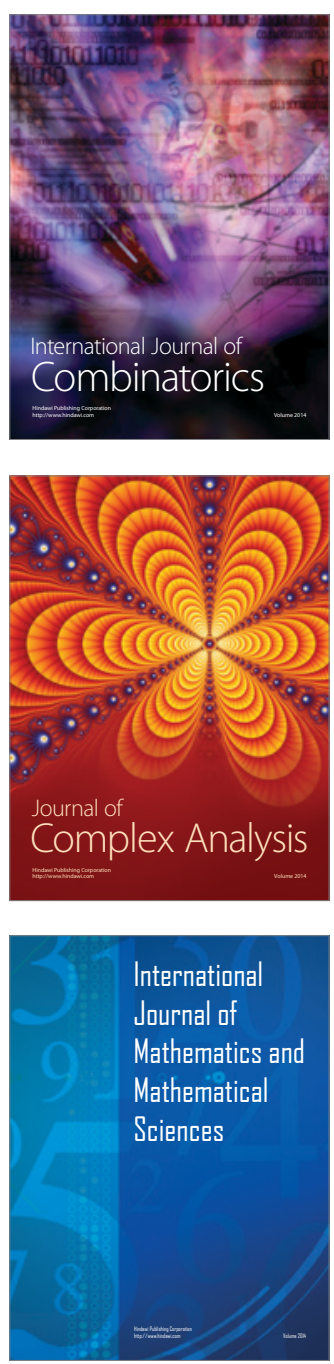
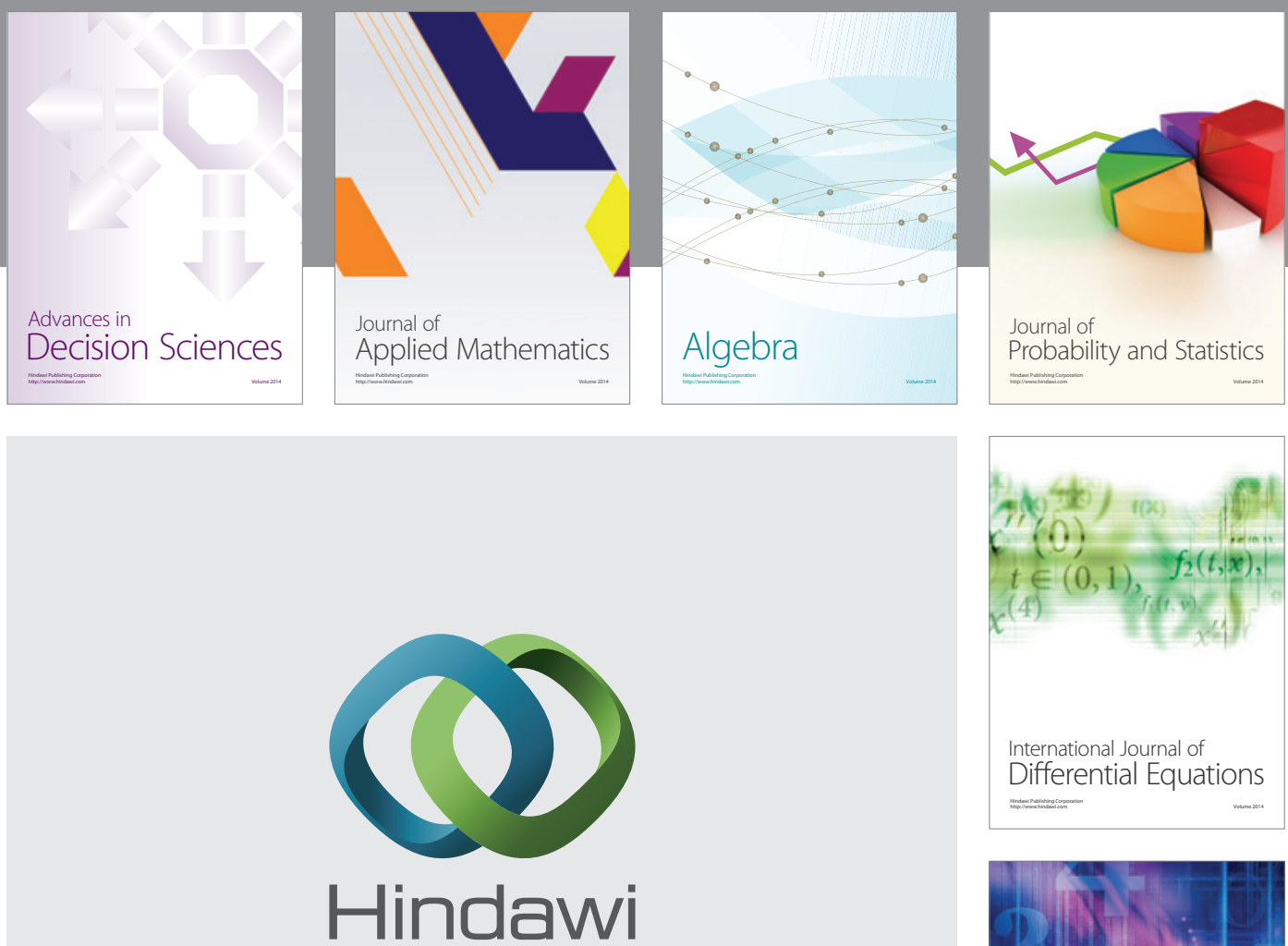

Submit your manuscripts at http://www.hindawi.com
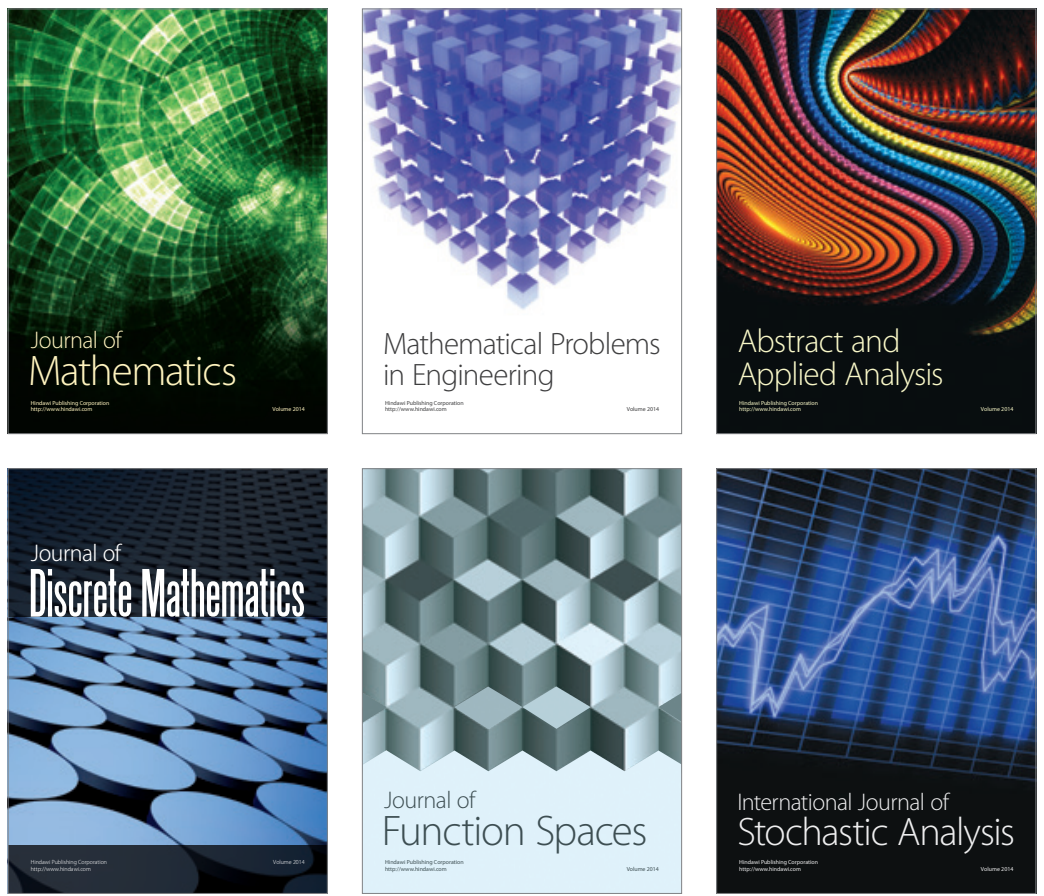

Journal of

Function Spaces

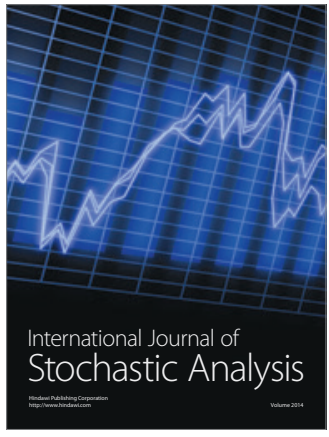

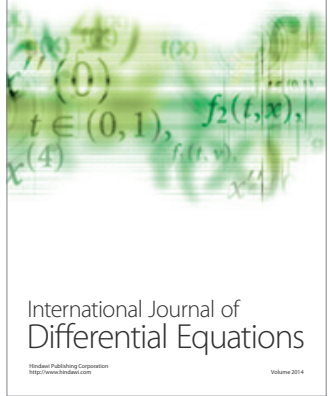
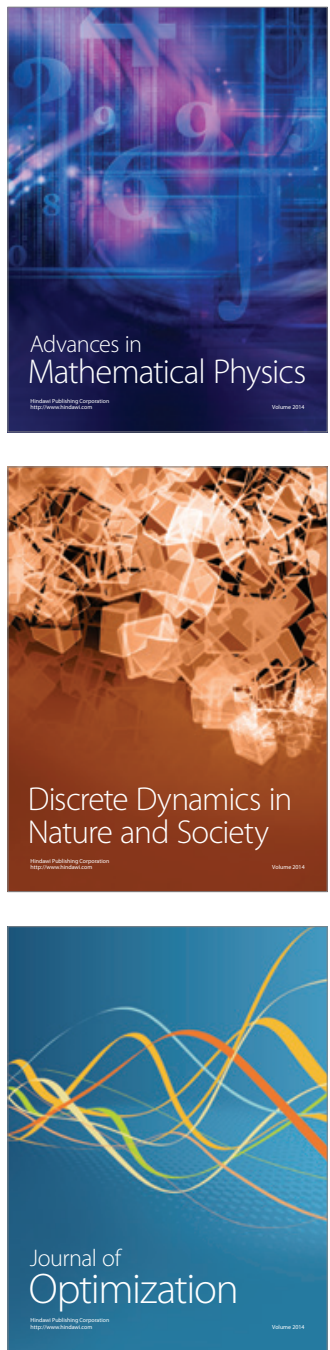